\title{
Nonlinear analysis of EEG in chess players
}

\author{
Pawel Stepien ${ }^{1 *}$, Wlodzimierz Klonowski ${ }^{1}$ and Nikolay Suvorov ${ }^{2}$
}

\author{
${ }^{*}$ Correspondence: \\ pstepien@ibib.waw.pl \\ ${ }^{1}$ Nalecz Institute of Biocybernetics \\ and Biomedical Engineering PAS, \\ Warsaw, Poland \\ Full list of author information is \\ available at the end of the article
}

\begin{abstract}
Background: The chess game is a good example of cognitive task which needs a lot of training and experience. The aim of this work is to compare applicability of two nonlinear methods - Higuchi Fractal Dimension and Empirical Mode Decomposition in analysis of EEG data recorded during chess match. We analyzed data of three master chess players registered during their matches with computer program.

Methods: We used two nonlinear methods: Higuchi Fractal Dimension that is a good and fast tool for analyzing signal complexity and modification of Empirical Mode Decomposition, called Sliding Window Empirical Mode Decomposition, that breaks down a signal into its monocomponents. Obtained results are compared with the resting state i.e. EEG during relax witch closed eyes.

Results: The analysis shows higher values of Higuchi Fractal Dimension during the thinking over chess moves than in the players' rest state. There are no statistically significant differences in contribution of EEG bands to total power of EEG calculated with Sliding Window Empirical Mode Decomposition.
\end{abstract}

Conclusions: Our results show beter applicability of Higuchi Fractal Dimension method for analysis of EEG signals related to chess tasks than that of Sliding Window Empirical Mode Decomposition.

Keywords: Chess; EEG; Higuchi fractal dimension; Fractal analysis; Empirical mode decomposition; Sliding window empirical mode decomposition

\section{Background}

The chess game, as expert task which needs a lot of training and experience, interested neuroscientists for a long time. For example Volke et al. (1998) [1] investigated 4 simple chess problems like: "pattern recognition" (if the King was or not on the board); if the King was in check; if the King was checkmated; checkmate in one move. They noticed that evoked coherences of EEG signals were sensitive both to sensory and mental activity (respectively theta and beta coherences), and beta coherences depended on the type of task. Later, Volke et al. (2002) [2] compared differences in evoked coherences between experts and novices - the experts showed higher values during tasks compared to the resting condition, whereas the novices - lower values.

The same chess problems were studied by Wright et al. (2013) [3]. They found differences in amplitudes of N2 and P3 components of event-related potential signals between professionals and beginners.

The game similar to chess, shogi, was studied by Wan et al. (2011) [4] with a functional magnetic resonance imaging (fMRI). They found two activations specific to experts: one

(C) 2015 Stepien et al.; licensee Springer on behalf of EPJ. This is an Open Access article distributed under the terms of the Creative Commons Attribution License (http://creativecommons.org/licenses/by/4.0), which permits unrestricted use, distribution, and reproduction in any medium, provided the original work is properly credited. 
in the precuneus of the parietal lobe during perception of board patterns, and the other in the caudate nucleus of the basal ganglia during quick generation of the best next move. Hänggi et al. (2014) [5] showed (with fMRI) that chess experts recruit different psychological functions and activate different brain areas during chess-related tasks: "Grey matter volume and cortical thickness were reduced in chess players compared with those of control men in the OTJ and precunei. The volumes of both caudate nuclei were not different between groups, but correlated inversely with the years of chess playing experience".

\section{Data}

Data of three chess master players: one of them is the Grandmaster (player A - 22 years old male) and others are the FIDE Masters (players B - 23 years old male and C - 22 years old male) were analyzed. The data were recorded by the group of Prof. Nikolay Suvorov (Institute of Experimental Medicine NWB RAMS, St.Petersburg, Russia) [6]. Players took the chess matches with computer program Fritz-10 (player A - two matches, B - two matches and $\mathrm{C}$ - one match). During (and before) each match the EEG signal from each player was recorded in 10-20 system (the following electrodes were used: Fp1, Fpz, Fp2, F7, F3, Fz, F4, F8, T3, C3, Cz, C4, T4, T5, P3, Pz, P4, T6, O1, Oz, O2 and A1, A2 as reference electrodes; sampling frequency was $500 \mathrm{~Hz}$ ). To avoid the artifacts players had closed eyes during the all match (and in control EEG recording before the play). The players had to store the actual arrangement on a chessboard in memory and they were informed about opponent's moves by the physician's voice. Every match was won by the computer program, and had about 40 moves and took $40 \pm 5$ minutes except first player A's first match which took 66 minutes.

EEG signal was filtered with band pass $0.53-150 \mathrm{~Hz}$ and with notch $45-55 \mathrm{~Hz}$ filter.

\section{Methods}

\section{Higuchi fractal dimension}

Higuchi Fractal Dimension (DF) [7] is calculated directly from the signal, in time domain, without reconstruction of strange attractors in multidimensional phase space. Value of $D F$ is always between 1 and 2 (simple curve has dimension equal 1 and a plane has dimension equal 2). Fractional part of $D F$ shows what fraction of the plane is "filled up" by the curve, so it is a measure of the signal's complexity. Further description of Higuchi Fractal Dimension is in Klonowski (2007) [8].

$D F$ of the resting state (relaxed with closed eyes) EEG record (about 250 seconds long) was calculated in 0.5 second length time windows and then averaged for each channel. $D F$ of EEG during the match was calculated at time intervals corresponding to the thinking on each chess movement by the player (it gave about 40 values of $D F$ for specific match) and then averaged for each channel. The maps of mean $D F$ for each channel were constructed. The standard deviation was used as a parameter of statistical significance.

\section{Empirical mode decomposition}

Empirical Mode Decomposition $(E M D)$ is an entirely data-driven algorithm which breaks down nonstationary, multicomponent signal into its monocomponents. Such monocomponents are called Intrinsic Mode Functions (IMFs). Each IMF must fulfill the following criteria: 

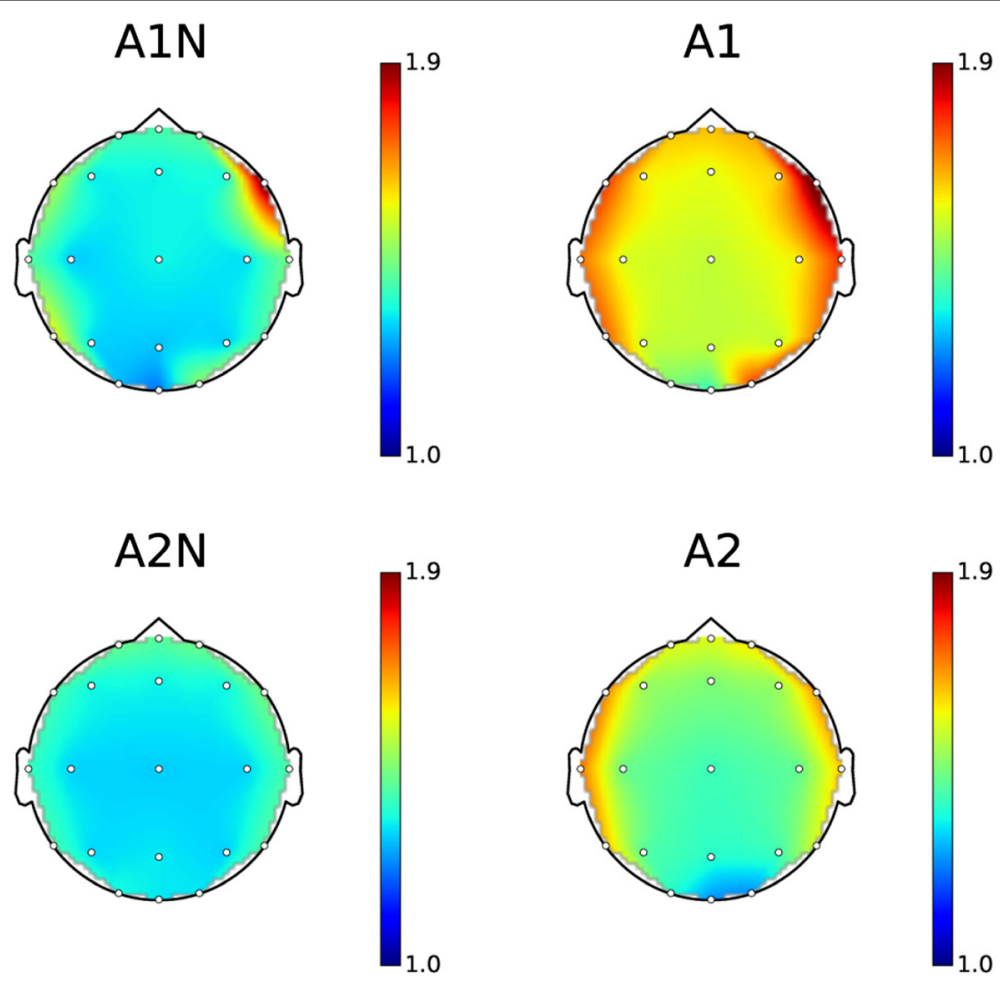

Figure 1 Mean $D F$ for player $A$ : before the first match (A1N); during the first match (A1); before the second match (A2N) and during the second match (A2).

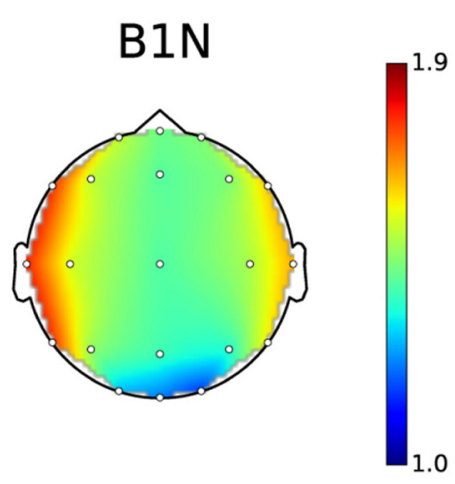

$\mathrm{B} 2 \mathrm{~N}$

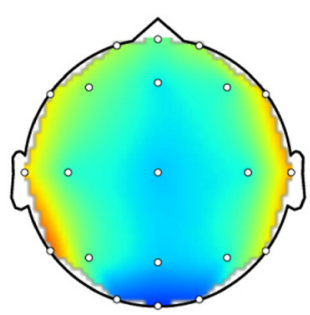

B1
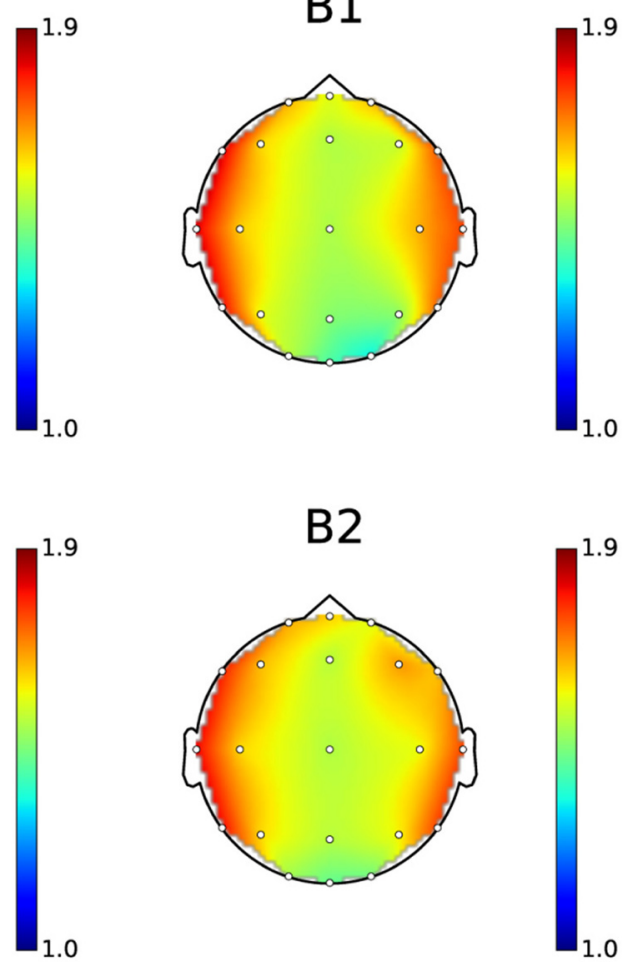

B2

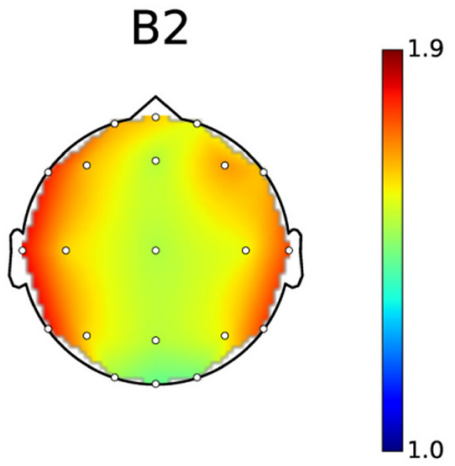

Figure 2 Mean $D F$ for player B: before the first match (B1N); during the first match (B1); before the second match (B2N) and during the second match (B2). 


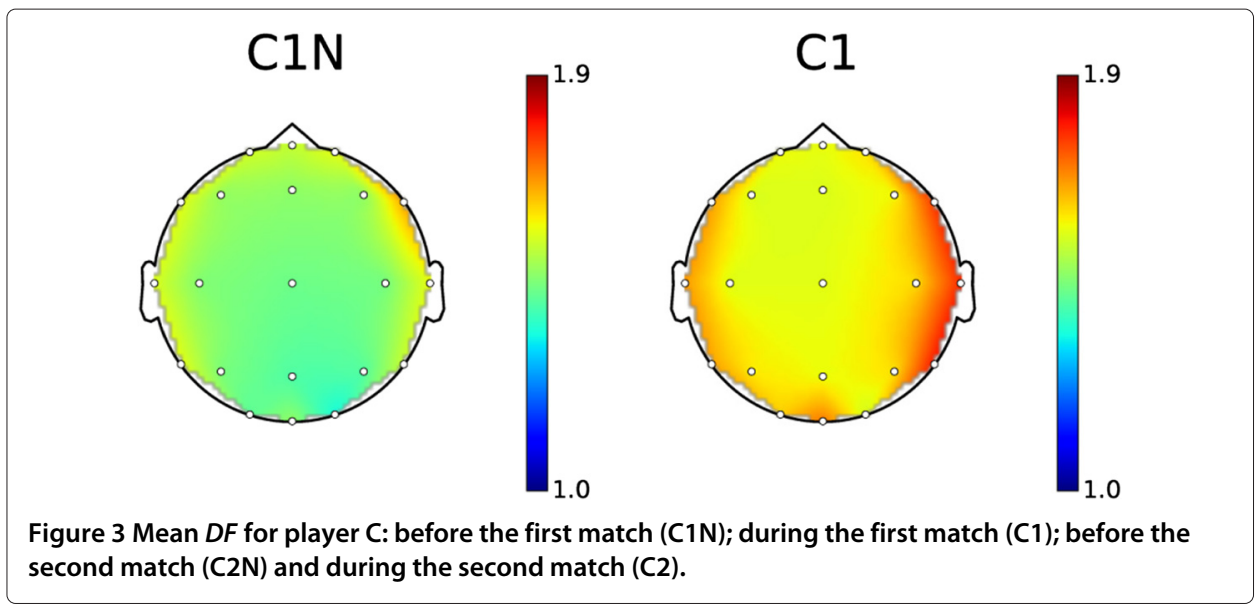

- the number of extrema and zeros are equal or their difference is not greater than 1 ,

- the signal has "zero mean" - the mean value of the envelope determined by maxima and the envelope determined by minima is equal 0 at every point.

Further description of $E M D$ is in Huang et al. (1998) [9]. We used modified algorithm called Sliding Window Empirical Mode Decomposition (SWEMD, see [10]) which speeds up the calculation about 10 times when compared with 'classical' $E M D$ method.

Signal decomposed by $S W E M D$ can be further analyzed by obtaining the marginal Hilbert-Huang Spectrum $h h s(f)$, see equation (17) in [10].

The information about the contribution of a frequency range to the total power of signal can be obtained by integration of the marginal Hilbert-Huang Spectrum with frequency

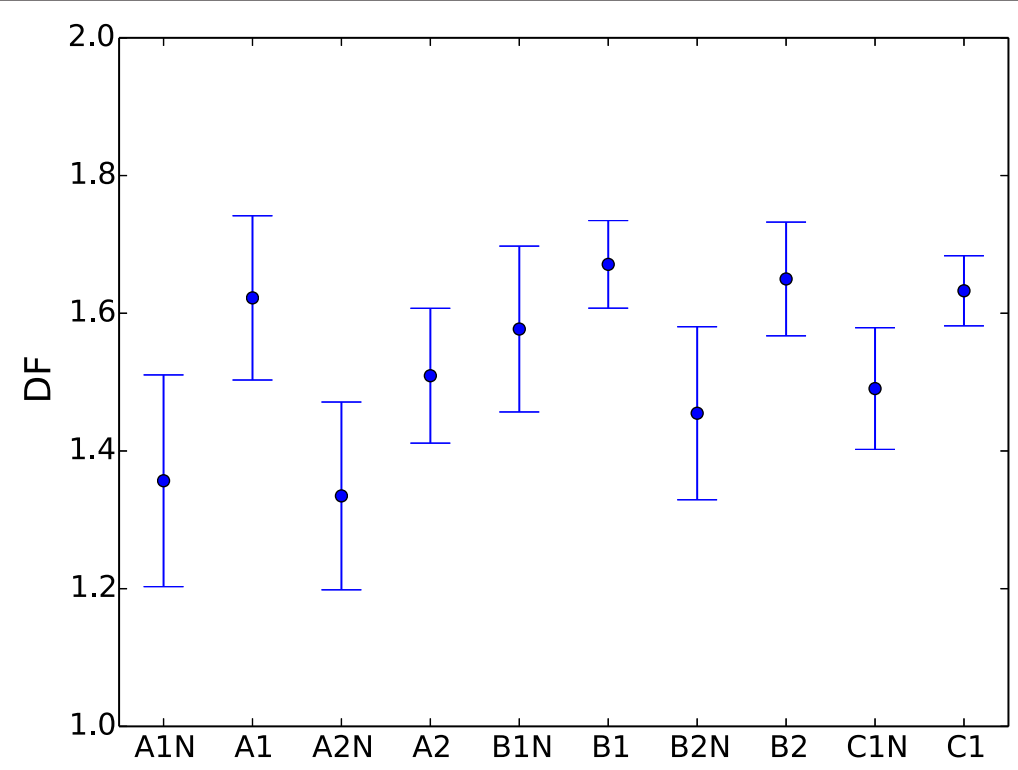

Figure 4 Mean $D F$ and standard deviation of EEG on electrodes $T 3, C 3, C z, C 4$ and $T 4$ before matches and during matches for all players. The difference of mean $D F$ is visible between matches and resting state for A1, B2 and C1 cases. 


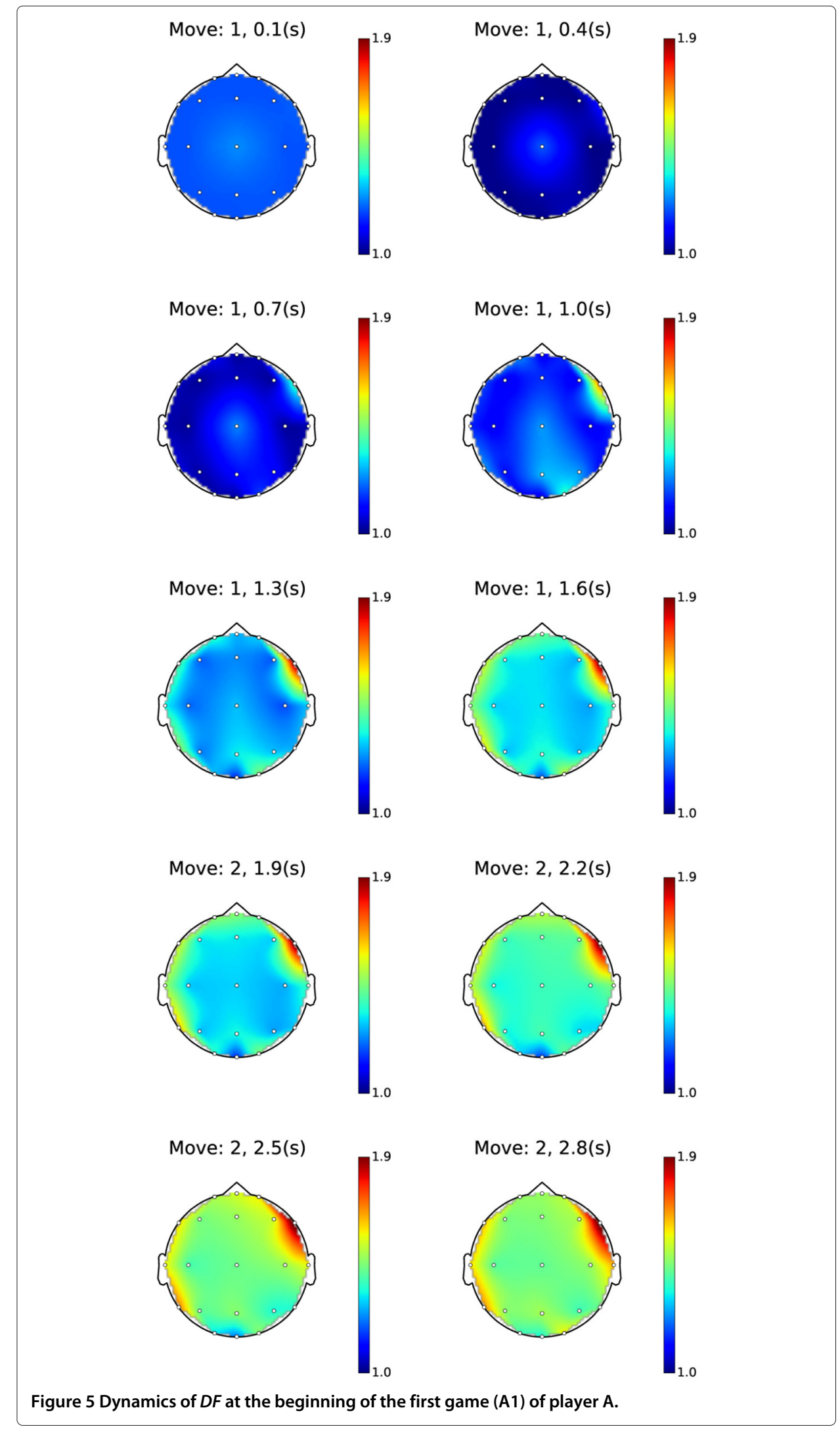


range limits. For example the contribution of the alpha band in EEG can be calculated with:

$$
\alpha \text { power }=\frac{\int_{8}^{13} h s s(f) d f}{\int_{0}^{\infty} h s s(f) d f}
$$

Contribution of each EEG band was calculated using SWEMD and the marginal HilbertHuang Spectrum in similar way as Higuchi Fractal Dimension. For the resting state contribution of each band was calculated in 0.5 second length time windows and then averaged for each channel and standard deviation was calculated. Bands' contributions during the match was calculated at time intervals corresponding to the thinking on each chess movement by the player and then averaged for each channel and standard deviation was obtained.

\section{Results}

Figures 1, 2 and 3 show (respectively for players A, B and C) differences in DF between the resting condition with closed eyes before the match and during the chess play. Figure 4 shows mean $D F$ and standard deviation of EEG on electrodes T3, C3, Cz, C4 and T4 before matches and during matches for all players.

Attached movie (Additional file 1) shows dynamics of Higuchi Fractal Dimension during the first match (A1) of player A (first ten moves). Interesting is the first move, when increasing of $D F$ is noticed. After about 2.5 seconds (Figure 5) the pattern of $D F$ becomes similar to Figure 1 A1.

Figures 6, 7, 8, 9 and 10 show mean contribution and standard deviation of each EEG (respectively: theta, delta, alpha, beta and gamma), calculated using SWEMD, on electrodes T3, C3, Cz, C4 and T4 before matches and during matches for all players.

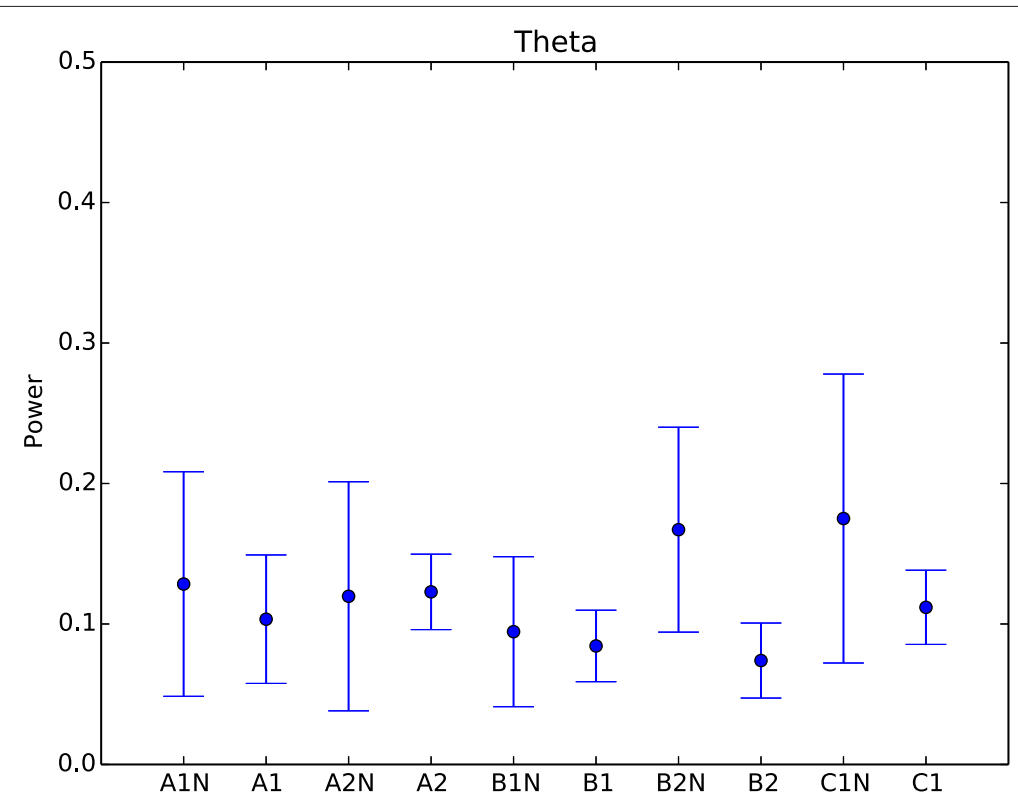

Figure 6 Mean contribution and standard deviation of theta EEG band on electrodes $T 3, C 3, C z, C 4$ and $\mathrm{T} 4$ before matches and during matches for all players. 


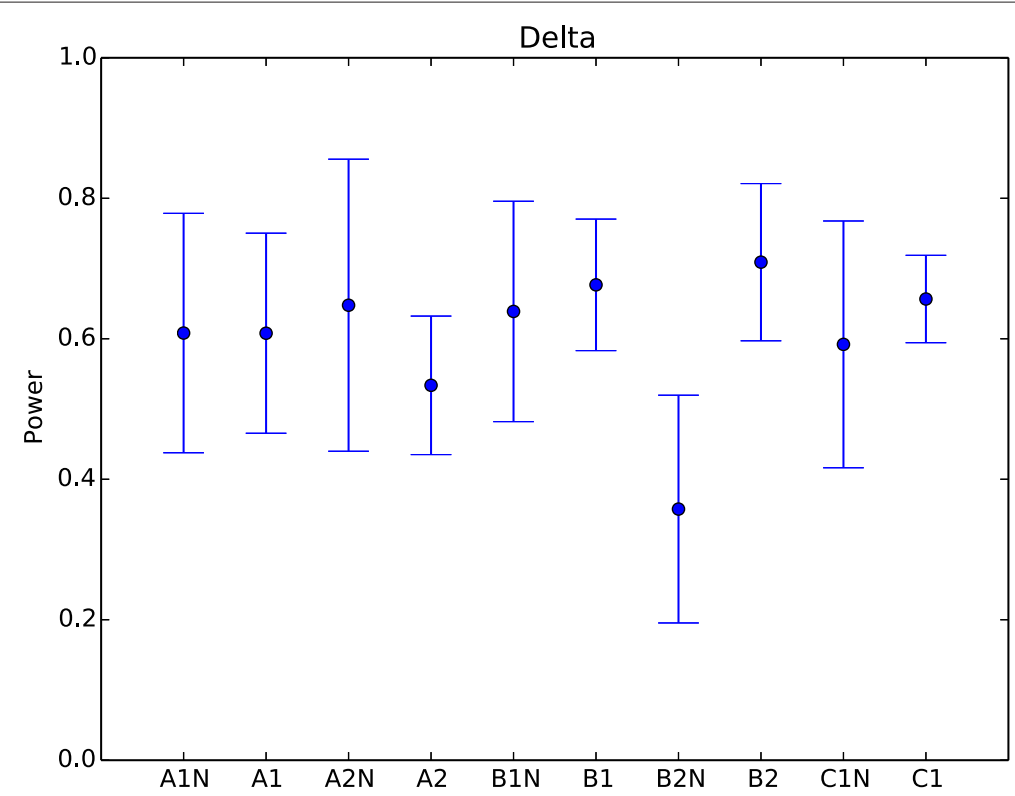

Figure 7 Mean contribution and standard deviation of delta EEG band on electrodes $\mathrm{T} 3, \mathrm{C} 3, \mathrm{Cz}, \mathrm{C} 4$ and $\mathrm{T} 4$ before matches and during matches for all players.

\section{Discussion}

The results show that $D F$ is higher during the match than in the resting state, mainly in lateral electrodes. Although this trend is clearly visible, it is not always statistically significant, in particular in games A2 and B1, as shown in Figure 4. Analysis of EEG bands' contributions, calculated with $S W E M D$, to total power of EEG gives no statistically significant differences between the resting state and chess match near in every case (Figures 6 , $7,8,9$ and 10).

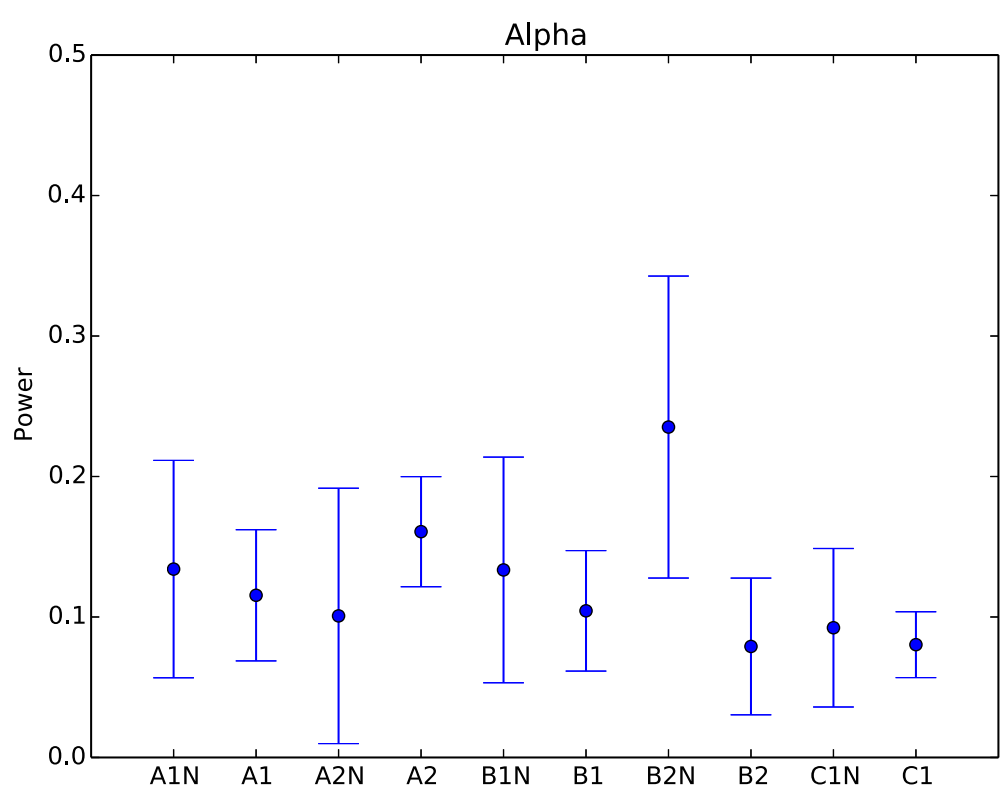

Figure 8 Mean contribution and standard deviation of alpha EEG band on electrodes $T 3, C 3, C z, C 4$ and $\mathrm{T} 4$ before matches and during matches for all players. 


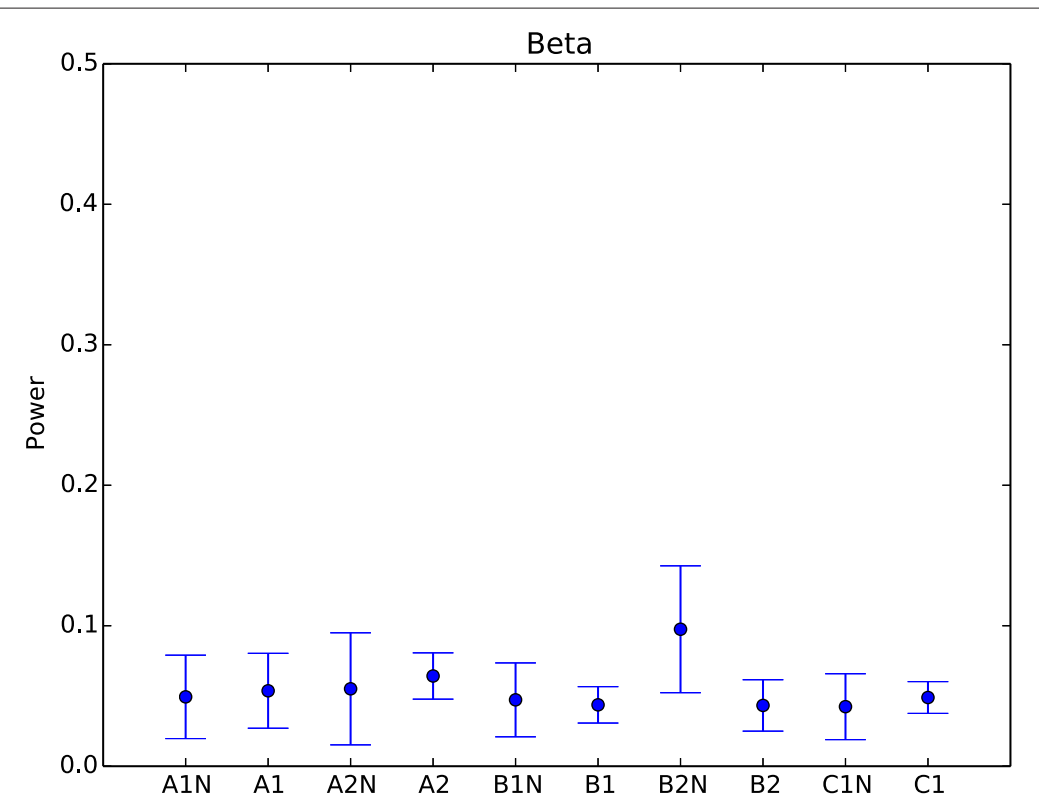

Figure 9 Mean contribution and standard deviation of beta EEG band on electrodes $\mathrm{T} 3, \mathrm{C} 3, \mathrm{Cz}, \mathrm{C} 4$ and $\mathrm{T} 4$ before matches and during matches for all players.

\section{Conclusions}

Presented results show better applicability of Higuchi Fractal Dimension method for analysis of EEG signals related to chess tasks than that of Sliding Window Empirical Mode Decomposition. DF shows that the EEG signal during the game is more complex, nonlinear, and non-stationary even when there are no significant differences between game and relaxed state in contribution of different EEG bands to total power of the signal. There

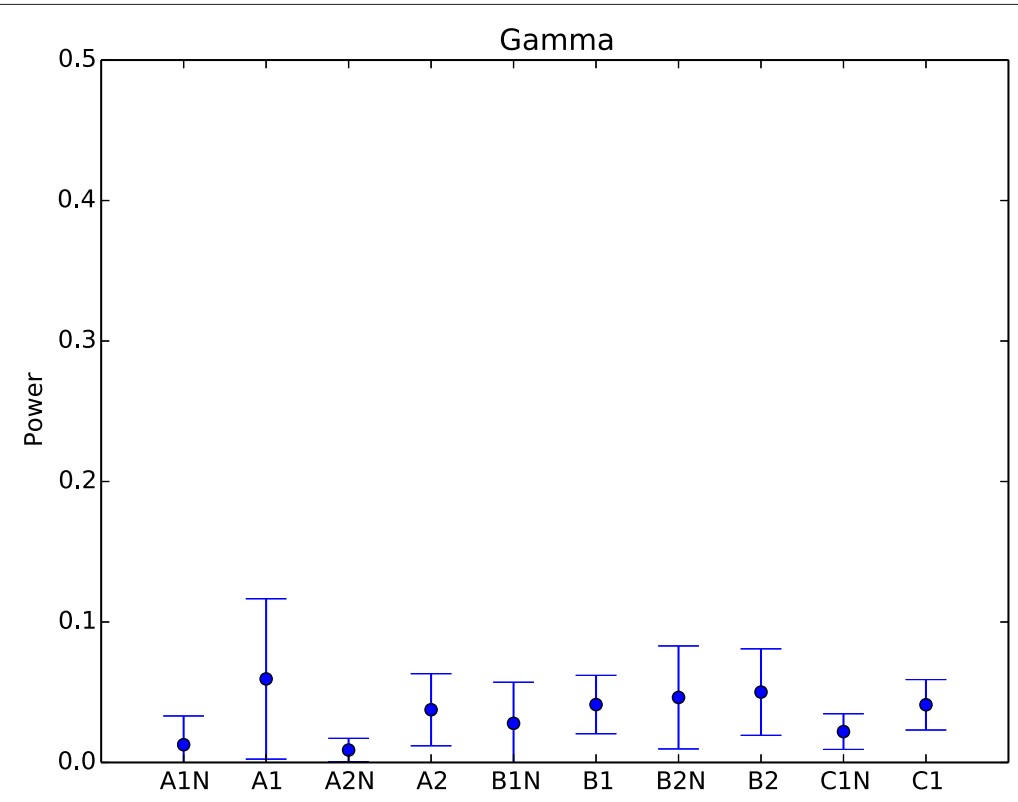

Figure 10 Mean contribution and standard deviation of gamma EEG band on electrodes $\mathrm{T} 3, \mathrm{C} 3, \mathrm{Cz}$, C4 and T4 before matches and during matches for all players. 
is the need of gathering more data from more chess experts and of comparing them with data from novice chess players.

\section{Additional file}

Additional file 1: Dynamics of Higuchi Fractal Dimension during the first match (A1) of player A (first ten moves of the match). The installed MPEG4 decoder is needed to open this movie.

\section{Competing interests}

The authors declare that they have no competing interests.

Authors' contributions

PS has performed the data analysis. PS and WK have written the paper. NS has provided data. All authors read and approved the final manuscript.

\section{Acknowledgements}

This work was supported by Nalecz Institute of Biocybernetics and Biomedical Engineering, Polish Academy of Sciences, Warsaw, through statutory project 4.4/st/14

\section{Author details}

${ }^{1}$ Nalecz Institute of Biocybernetics and Biomedical Engineering PAS, Warsaw, Poland. ${ }^{2}$ Institute of Experimental Medicine NWB RAMS, St. Petersburg, Russia.

Received: 26 August 2014 Accepted: 12 January 2015

Published online: 12 March 2015

\section{References}

1. Volke H-J, Dettmar P, Richter P, Rudolf M. Evoked coherences of eeg in chess playing. Int J Psychophysiol. 1998:30(1-2):225-6.

2. Volke H-J, Dettmar P, Richter P, Rudolf M, Buhss U. On-coupling and off-coupling of neocortical areas in chess experts and novices as revealed by evoked eeg coherence measures and factor-based topological analysis-a pilot study. J Psychophysiol. 2002;16(1):23.

3. Wright MJ, Gobet F, Chassy P, Ramchandani PN. Erp to chess stimuli reveal expert-novice differences in the amplitudes of n2 and p3 components. Psychophysiology. 2013;50(10):1023-33.

4. Wan X, Nakatani H, Ueno K, Asamizuya T, Cheng K, Tanaka K. The neural basis of intuitive best next-move generation in board game experts. Science. 2011;331(6015):341-6.

5. Hänggi J, Brütsch K, Siegel AM, Jäncke L. The architecture of the chess player's brain. Neuropsychologia. 2014;62(0): $152-62$

6. Suvorov NB, Bozhokin SV, Polonski YuZ. Electrophysiological correlates of human intellectual activity during a chess-game with the computer program blindly. Wavelet analysis. Inf Control Syst. 2012;3:71-76.

7. Higuchi T. Approach to an irregular time series on the basis of the fractal theory. Physica D: Nonlinear Phenom. 1988:31(2):277-83.

8. Klonowski W. From conformons to human brains: an informal overview of nonlinear dynamics and its applications in biomedicine. Nonlinear Biomed Phys. 2007;1(1):5

9. Huang NE, Shen Z, Long SR, Wu MC, Shih HH, Zheng Q, et al. The empirical mode decomposition and the Hilbert spectrum for nonlinear and non-stationary time series analysis. Proc R Soc London. Ser A: Math Phys Eng Sci. 1998:454(1971):903-95.

10. Stepien P. Sliding window empirical mode decomposition - its performance and quality. EPJ Nonlinear Biomed Phys. 2014;2(1):14 